\title{
A Novel Strategy for Fabricating a Strong Nanoparticle Monolayer and Its Enhanced Mechanism
}

\author{
Jun Zhou ${ }^{1, *}$, Xiaoqing Cao ${ }^{1}$, Linlin $\mathrm{Li}^{2}$, Xingcheng Cui ${ }^{2}$ and $\mathrm{Yu} \mathrm{Fu}{ }^{2} \mathbb{D}$ \\ 1 Key Laboratory for Anisotropy and Texture of Materials, School of Materials Science and Engineering, \\ Northeastern University, Shenyang 110819, China; caoxiaoqingfly@163.com \\ 2 College of Sciences, Northeastern University, Shenyang 110819, China; lilinlin211@163.com (L.L.); \\ NEUcuixingchen@163.com (X.C.); fuyu@mail.neu.edu.cn (Y.F.) \\ * Correspondence: hafouniu@163.com
}

Received: 20 September 2019; Accepted: 13 October 2019; Published: 16 October 2019

\begin{abstract}
This work presents the preparation of cross-linking Au nanoparticle (NP) monolayer membranes by the thiol exchange reaction and their enhanced mechanical properties. Dithiol molecules were used as a cross-linking mediator to connect the adjacent nanoparticles by replacing the original alkanethiol ligand in the monolayer. After cross-linking, the membrane integrity was maintained and no significant fracture was observed, which is crucial for the membrane serving as a nanodevice. TEM (Transmission Electron Microscopy), UV-Vis absorption spectrum, and GISAXS (grazing incidence small angle X-ray scattering) were performed to characterize the nanostructure before and after cross-linking. All results proved that the interparticle distance in the monolayer was controllably changed by using dithiols of different lengths as the cross-linking agent. Moreover, the modulus of the cross-linking monolayer was measured by atomic force microscopy (AFM) and the result showed that the membrane with a longer dithiol molecule had a larger modulus, which might derive from the unbroken and intact structure of the cross-linking monolayer due to the selected appropriately lengthed dithiol. This study provides a new way of producing a nanoparticle monolayer membrane with enhanced mechanical properties.
\end{abstract}

Keywords: gold; nanoparticles; self-assembly; monolayer; cross-linking

\section{Introduction}

Freestanding nanoparticle (NP) ultra-thin membranes as a novel nanomaterial have been applied in various fields including integrated microcircuits, chemical sensors, and so on [1-9]. The multilayered structure of the membrane causes difficulties in studying its fundamental properties. Thus, research interest has led to a monolayer membrane due to its simple two-dimension structure and abundant physicochemical properties that can be easily regulated by the interaction between adjacent nanoparticles $[10,11]$. The NP monolayer membrane has been regarded as an appropriate object for fundamental research and has a good prospective in various applications.

The mechanical properties are crucial for the monolayer membrane to function in the long-term, especially when serving as a basic unit to fabricate integrated components or nano-devices [12-14]. Generally, the NPs monolayer membranes fabricated by traditional methods have a low ordering degree and weak interaction among nanoparticles. Therefore, such structured membranes are fragile and difficult to employ [15]. In addition, the low-ordering pattern in the membrane and the poor mechanical properties limit the area of fabricated membrane that is required for many special applications. So far, numerous methods have been developed to improve the mechanical properties of a NP monolayer membrane based on physical and chemical technology [16-19]. For instance, slightly heating the 
$\mathrm{Au}$ NPs self-assembled at the water-alcohol interface produced a firm and large (several square centimeters) freestanding monolayer [16]. The acquired monolayer membrane showed an obvious promotion in mechanical strength and presented a high structure stability. However, the ordering degree of the monolayer was sacrificed after heat treatment because of the fusion of some particles, leading to a change in geometric configuration and deteriorated physiochemical properties such as thermal conductivity. The strength of the monolayer can also be enhanced by applying chemistry methods such as using cross-linking to enhance the interaction between nanoparticles [17-19]. A typical cross-linking agent is the dithiol molecule containing the -SH group. Taking advantage of the strong $\mathrm{Au}-\mathrm{S}$ bonding, the dithiol links adjacent nanoparticles together tightly and subsequently strengthens the nanomembrane [20]. Kowalczyk et al. utilized an alkane dithiol molecule chemically crosslinked NPs monolayer membrane that showed high stability against the corrosion of organic solvents. As a result, the membrane could be deposited onto various topography surfaces while maintaining its own structure [21]. Though effective to improve the strength, the cross-linking method starts from the monolayer of disordered nanoparticles. The random distribution of Au nanoparticles in the monolayer membrane deteriorates the electrical conductance and optical property, limiting its applications. In order to solve this problem, a monolayer of ordered NPs was introduced into the cross-linking reaction $[22,23]$. The dithiol molecules replaced the capping molecules on the surface of the nanoparticles by the exchange reaction and successfully linked adjacent nanoparticles [22]. However, most exchange reactions are performed in a harsh solution for ordered structure. In this scenario, offering a relatively rigid monolayer with ordered structure is necessary. We prepared a stable ordered NP monolayer by vacuum thermal treating a self-assembled Au NP monolayer [11,24,25]. In this process, the alkyl chains of the ligand molecule generate a deep interdigitation with each other, leading to a strong interaction between the nanoparticles and a good resistance against various solvents.

Based on this, we present the preparation of a Au monolayer using a thermally treated Au NP ordered monolayer. To do this, various dithiol molecules with different alkyl chain lengths were applied to replace the original ligand molecules by thee exchange reaction. TEM (Transmission Electron Microscopy) and GISAXS (grazing incidence small angle X-ray scattering) characterization demonstrated that the alkyl chain length of the dithiol molecule could effectively tune the interparticle spacing in the monolayer. Quantitative measurement of the cross-linking membrane by AFM (Atomic Force Microscope) showed that their Young's modulus gradually increased from the original $204.5 \mathrm{MPa}$ to $268.5 \mathrm{MPa}$, signifying the role that chain length plays in determining the mechanical strength of the monolayer. To our best knowledge, this is the first report on the strengthening mechanism of crosslinked membranes.

\section{Materials and Methods}

\subsection{Materials and Instruments}

Chloroauric acid $\left(\mathrm{HAuCl}_{4} \cdot 4 \mathrm{H}_{2} \mathrm{O}\right)$, sodium borohydride $\left(\mathrm{NaBH}_{4}\right)$, toluene $\left(\mathrm{C}_{7} \mathrm{H}_{8}\right)$, acetone $\left(\mathrm{C}_{3} \mathrm{H}_{6} \mathrm{O}\right)$, n-hexane $\left(\mathrm{C}_{6} \mathrm{H}_{14}\right)$, and alcohol $\left(\mathrm{C}_{2} \mathrm{H}_{6} \mathrm{O}\right)$ were obtained from Sinopharm Chemical Reagent Co. Ltd. (Shenyang, Liaoning, China) and were used without further purification. $1 \mathrm{H}, 1 \mathrm{H}, 2 \mathrm{H}$, 2H-perfluorodecyltriethoxysilane $\left(\mathrm{C}_{16} \mathrm{H}_{19} \mathrm{~F}_{17} \mathrm{O}_{3} \mathrm{Si}\right)$, 1-dodecanethiol $\left(\mathrm{C}_{12} \mathrm{H}_{26} \mathrm{~S}\right)$, 1,2-ethanedithiol $\left(\mathrm{C}_{2} \mathrm{H}_{6} \mathrm{~S}_{2}\right)$, 1,3-dimercaptopropane $\left(\mathrm{C}_{3} \mathrm{H}_{8} \mathrm{~S}_{2}\right)$, and 1,6-hexanedithiol $\left(\mathrm{C}_{6} \mathrm{H}_{14} \mathrm{~S}_{2}\right)$ were purchased from Alfa Aesar (Ward Hill, MA, USA). 1,4-Butanedithiol $\left(\mathrm{C}_{4} \mathrm{H}_{10} \mathrm{~S}_{2}\right)$ and 1,5-pentanedithiol $\left(\mathrm{C}_{5} \mathrm{H}_{12} \mathrm{~S}_{2}\right)$ were acquired from Tokyo Kasei Kogyo Co., Ltd. (Tokyo, Japan) and were used as received. Teflon tape was purchased from SPi Supplies (USA). Grazing incidence small angle x-ray scattering (GISAXS, Shanghai, China) was carried on the Shanghai Synchrotron Radiation Facility (SSRF, Shanghai, China). Atomic force microscopy (AFM, Karlsruhe, Germany) was measured on a Nanosurf Easyscan 2. Transmission electron microscope (TEM) observations were performed on a JEOL JEM-2100F (Tokyo, Japan). 


\subsection{Methods}

\subsubsection{Preparation and Phase Transfer of Au NPs}

The Au NPs were prepared following the literature based on $\mathrm{NaBH}_{4}$ as a reduction agent [26]. First, $9.475 \mathrm{~mL}$ deionized water together with $95 \mu \mathrm{L} \mathrm{HAuCl} / \mathrm{HCl}$ mixture $\left(50 \mathrm{mmol} \cdot \mathrm{L}^{-1}\right)$ were added into a $50 \mathrm{~mL}$ colorimetric tube under the vibration of a vortex mixer. Then, $425 \mu \mathrm{L} \mathrm{NaOH} / \mathrm{NaBH} 4$ solution $\left(50 \mathrm{mmol} \cdot \mathrm{L}^{-1}\right)$ was injected into the $\mathrm{HAuCl}_{4}$ mixture immediately, followed by immersing the colorimetric tube into boiling water for $2.5 \mathrm{~min}$, before finally the Au NPs solution was obtained. To transfer the Au NPs from water to hexane, 1-dodecanethiol mixed with n-hexane was used to exact the Au NPs by shaking the solution for about $30 \mathrm{~s}$. After that, the hexane phase turned dark red from colorless, which indicated that the Au NPs had transferred to the hexane successfully.

\subsubsection{Fabrication of Ultrathin Monolayer Au NP Membranes on Quartz Plate and Heat Treatment}

Before the experiment, quartz plates $(2 \times 2 \mathrm{~cm})$ were immersed into an ethanol solution and cleaned with absorbent cotton repeatedly, followed by soaking for $30 \mathrm{~min}$ in n-hexane. After ultrasonic cleaning for $10 \mathrm{~min}$, the quartz plates were treated with plasma bombardment to remove organic pollutants and were then placed vertically into a regent bottle with $4 \mu \mathrm{L}$ perfluorinated regent $(1 \mathrm{H}, 1 \mathrm{H}$, $2 \mathrm{H}, 2 \mathrm{H}$-perfluorodecyltriethoxysilane). Next, the bottles with quartz substrates were heated for $5 \mathrm{~h}$ at $60{ }^{\circ} \mathrm{C}$. The perfluorinated regent would volatilize and absorb on the surface of the substrates to form a perfluorinated monolayer, meaning the transformation of the surface of the quartz plates to go from hydrophilic to hydrophobic.

The fabrication of the Au NP monolayer membranes was as follows. Briefly, $150 \mu \mathrm{L}$ of toluene was first dropped on the pretreated quartz plates and the Au NPs solution in hexane was dropped to the toluene gradually until the drop surfaces were completely covered with NPs. After the solution volatilized completely, a close-packed Au NP monolayer membrane was obtained. In this experiment, part of the Au NP arrays was heated for another $5 \mathrm{~h}$ at $80^{\circ} \mathrm{C}$ under $200 \mathrm{~Pa}$.

\subsubsection{Fabrication of Ultrathin Monolayer Au NP Membranes on Teflon Tape and Heat Treatment}

Prior to use, the tape surface was treated by toluene wiping and dried in a vacuum oven for $30 \mathrm{~min}$ at $80^{\circ} \mathrm{C}$, which makes it able to retain a clean surface.

This assembly process was similar to the one described above. A close-packed Au NP monolayer was prepared on the tape surface. Additionally, one half of the Au NP membranes was put into centrifuge tubes, followed by heat treatment in a vacuum oven at $80^{\circ} \mathrm{C}$ for $0.5 \mathrm{~h}$ under $200 \mathrm{~Pa}$.

\subsubsection{Cross-Linking of Ultrathin Monolayer Au NP Membranes}

The ligand exchange experiments were performed in an alcohol solution. Typically, a Au NP array prepared on two different substrates was immersed into a solution with $20 \mathrm{mmol} \cdot \mathrm{L}^{-1}$ of the cross-linking agent away from light about $12 \mathrm{~h}$ (different cross-linking agents are listed in Table 1). After that, the Au NPs array was washed carefully with alcohol and dried in air. The obtained array was measured by UV-Vis (range $=400 \sim 900 \mathrm{~nm}$, speed $=0.5 \mathrm{~nm}$, maximum absorbance $=2.000 \mathrm{Abs}$.) and TEM. GISAXS $\left(\lambda=1.24 \mathrm{~nm}\right.$, incident angel $=0.2^{\circ}$, reflex angle $\left.=0.4^{\circ}\right)$ was also applied to obtain information about the change in interparticle distance.

Table 1. Cross-linking ligands employed in the exchange reaction.

\begin{tabular}{ccc}
\hline Types of Ligands & Concentrations $\left(\mathbf{m m o l}^{\left.-\mathbf{L}^{-1}\right)}\right.$ & Volume $(\boldsymbol{\mu} \mathbf{L})$ \\
\hline 1,2-ethanedithiol $\left(\mathrm{C}_{2} \mathrm{H}_{6} \mathrm{~S}_{2}\right)$ & 20 & 17 \\
1,3-dimercaptopropane $\left(\mathrm{C}_{3} \mathrm{H}_{8} \mathrm{~S}_{2}\right)$ & 20 & 20 \\
1,4-Butanedithiol $\left(\mathrm{C}_{4} \mathrm{H}_{10} \mathrm{~S}_{2}\right)$ & 20 & 23 \\
1,5-pentanedithiol $\left(\mathrm{C}_{5} \mathrm{H}_{12} \mathrm{~S}_{2}\right)$ & 20 & 27 \\
1,6-hexanedithiol $\left(\mathrm{C}_{6} \mathrm{H}_{14} \mathrm{~S}_{2}\right)$ & 20 & 30 \\
\hline
\end{tabular}




\section{Results and Discussion}

\subsection{Preparation of Au NP Membranes and Heat Treatment}

The Au NP membranes were prepared in accordance with the reported methods [26]. The detailed fabrication process is illustrated in Scheme 1. Prior to the experiment, a perfluorinated reagent was used to modify the quartz substrates in order to obtain a hydrophobic surface by the silane coupling reaction. After that, a drop of toluene was first dropped onto the substrate carefully and then Au NPs were spread on toluene to self-assemble at the toluene-air interface. After the toluene solution evaporated completely, a well-ordered Au NP monolayer array was deposited on the quartz substrate. TEM characterization results showed that the nanoparticles were close-packed to each other and the array presented a light pink without any visible wrinkle and breakage, as shown in Figure 1A. This phenomenon demonstrates that the prepared Au NP array possessed a regular internal structure and uniform external morphology. This assembly method is simple and highly reproducible, once it has satisfied the assembly conditions, which is the charged particles and an appropriate ligand molecule on the surface of the nanoparticles. The prepared gold nanoparticles are easily self-assembly at the toluene-air interface due to the equilibrium interaction deriving from electrostatic force and van der Waals forces. We believe that this method could be extended to other nanoparticles only if meeting the self-assembly condition on the toluene-air interface.

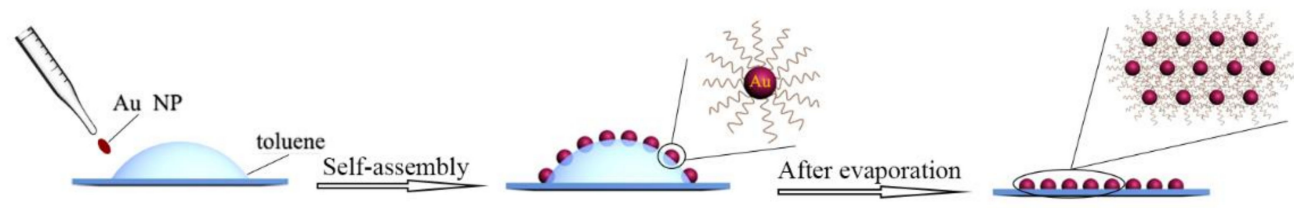

Scheme 1. Schematic of the self-assembly process of the Au nanoparticles.
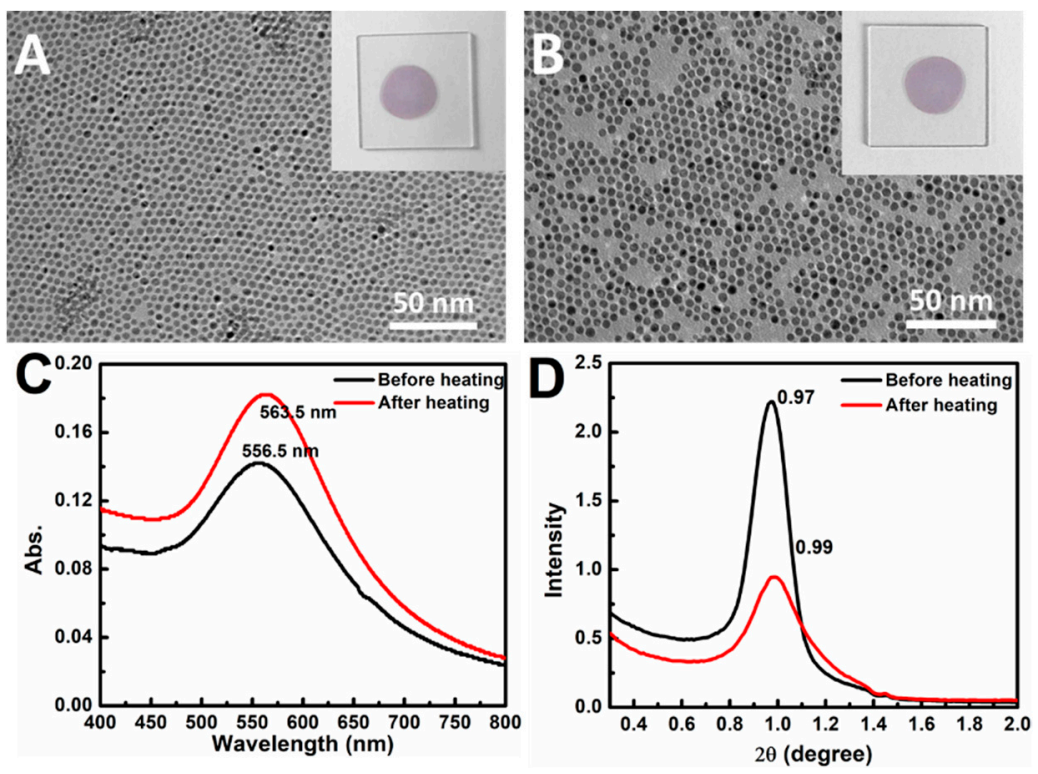

Figure 1. TEM images of the Au NP array fabricated on the quartz substrate (A) before and (B) after heating (inset is the optical photograph of array); (C) UV-Vis absorption spectrum; (D) SAXS (small angle X-ray scattering) curves of the Au NP array fabricated on the quartz substrate.

In order to enhance the monolayer stability, the prepared Au NP array was treated by vacuum heating at $80{ }^{\circ} \mathrm{C}$ for $0.5 \mathrm{~h}$ under $200 \mathrm{~Pa}$. As shown in Figure 1B, the color of the Au NP array changed from red to purplish red and the packing of the particles became closer. Correspondingly, the UV-Vis measurement shows the maximum absorption wavelength $\left(\lambda_{\max }\right)$ of the Au NP array's red shift from 
$556.5 \mathrm{~nm}$ to $563.5 \mathrm{~nm}$ (Figure 1C), suggesting the enhanced surface plasma resonance (SPR) effect. This phenomenon might be derived from the decreased interparticle spacing, which is consistent with the results from the TEM characterization. Additionally, the sample was measured by SAXS, as shown in Figure 1D. It is obvious that the grazing angle increased from 0.97 to 0.99 along with the broaden peak shape after the heat treatment, indicating a decreased lattice constant and reduced structure order in the array. According to the reported literature [11], the NP interparticle distance (D) can be calculated by the following formulas (Equations (1)-(4)):

$$
\begin{gathered}
\mathrm{D}=2 \pi / \mathrm{q}_{\mathrm{y}} \\
\mathrm{q}=\left(\mathrm{q}_{\mathrm{x}}^{2}+\mathrm{q}_{\mathrm{z}}{ }^{2}\right)^{1 / 2}, \\
\mathrm{q}_{\mathrm{x}}=4 \sin \left(\theta_{\mathrm{x}}\right) / \lambda, \\
\mathrm{q}_{\mathrm{z}}=4 \sin \left(\theta_{\mathrm{z}}\right) / \lambda,
\end{gathered}
$$

where $\mathrm{D}$ is the interparticle distance; $\mathrm{q}$ represents the diffraction vector; $\theta_{\mathrm{x}}$ is from the peak value; and $\theta_{\mathrm{Z}}\left(0.2^{\circ}\right)$ is obtained from the reflex angle. During the calculated process, $\mathrm{q}_{\mathrm{x}}$ was replaced by the $\mathrm{q}$. Here, the interparticle distance changed from $2.66 \mathrm{~nm}$ to $2.55 \mathrm{~nm}$ after heat treatment. All of the above results demonstrate that the interparticle distance is decreased by heat treatment. According to our previous report [24], the reduced distance between adjacent nanoparticles facilitated the interdigitation of the alkyl chain ligand and led to an improvement in the stability of the Au NP array.

\subsection{Cross-Linking of Au NP Membranes}

In order to obtain the cross-linking nanoparticles array, the preheated Au NPs array was immersed into an alcohol solution of dodecanethiolate (including 1,2-ethanedithiol $\left(\mathrm{C}_{2}\right), 1,3$-dimercaptopropane $\left(C_{3}\right)$, 1,4-butanedithiol $\left(C_{4}\right)$, 1,5-pentanedithiol $\left(C_{5}\right)$, and 1,6-hexanedithiol $\left(C_{6}\right)$ ) to trigger an exchange reaction. In this process, the 1-dodecanethiol ligand was replaced gradually by the dithiol molecule due to the concentration effect in solution. As shown in Figure 2, the cross-linking arrays almost maintained their integrity without any obvious destruction in the macroscopic view. Compared with the preheated array, the cross-linking array presented a different color that changed from bluish violet to green, along with the decreased dodecanethiolate length. We concluded that the neighboring nanoparticles were dragged to approach each other by the short dithiol molecule, which resulted in a reduced interparticle spacing and enhanced LSPR (Localized Surface Plasmon Resonance) (representing the color change). This conclusion was also proven by the UV-Vis absorption spectrum measurement, as shown in Figure 3F. The $\lambda_{\max }$ of the Au NP array red shifted gradually with the decreased carbon numbers $\left(C_{2}\right.$ to $\left.C_{8}\right)$. Detailed information about the $\lambda_{\max } v s$ carbon numbers is shown in the inset of Figure 3F. It is obvious that $\lambda_{\max }$ changed from $567 \mathrm{~nm}$ to $628 \mathrm{~nm}$ with the ligand changing from 1,8-hexanedithiol $\left(\mathrm{C}_{8}\right)$ to 1,2-ethanedithiol $\left(\mathrm{C}_{2}\right)$. These results demonstrate that the ligand exchange had been achieved and 1-dodecanethiol was successfully replaced by the dithiol molecule, which had a favorable regulation effect for the interparticle distance. Notably, the $\lambda_{\max }$ of the Au NP array and the carbon numbers $\left(C_{2}-C_{8}\right)$ of the dithiol molecule presents a linear correlation. According to the literature, the length for the 1,6-hexanedithiol molecules was about $1.5 \mathrm{~nm}$, which was nearly equal to the interparticle distance in the pre-heated Au NP array [10,26]. For the 1,8-octanedithiol, the long chain will not affect the array structure because of their flexibility property. Therefore, the exchange between the dithiol and 1-dodecanethiol hardly changed the interparticle distance, and hence would well maintain the structure of the Au NP array and LSPR property.

To further investigate the cross-linking array, TEM characterization was performed. As shown in Figure 3A-E, the array still remained as a single layer structure after cross-linking. Compared with the origin of the Au NP array, the interparticle distance of the crosslinked Au NP array decreased gradually, even appearing as a dumbbell structure $\left(C_{2}\right)$ with the reduced alkyl chain length of dithiol molecule. Meanwhile, the defect in the array begins to form and even generates a big cavity when a shorter dithiol 
is employed. All these results were consistent with the UV-Vis measurement, demonstrating the cross-linking reactions and their regulation effect for the interparticle spacing and array microstructure.
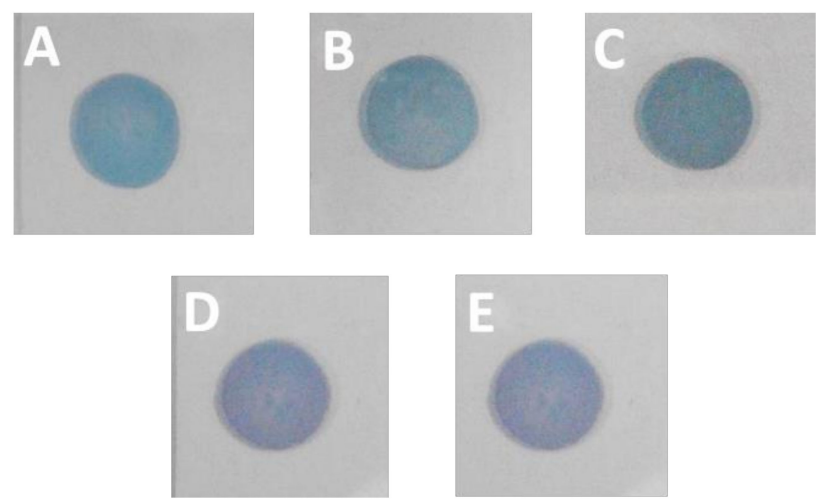

Figure 2. Photo images of the Au NP array cross-linked with ligands: (A) 1,2-ethanedithiol; (B) 1,3-dimercaptopropane; (C) 1,4-butanedithiol; (D) 1,5-pentanedithiol; (E) 1,6-hexanedithiol on the quartz substrate.
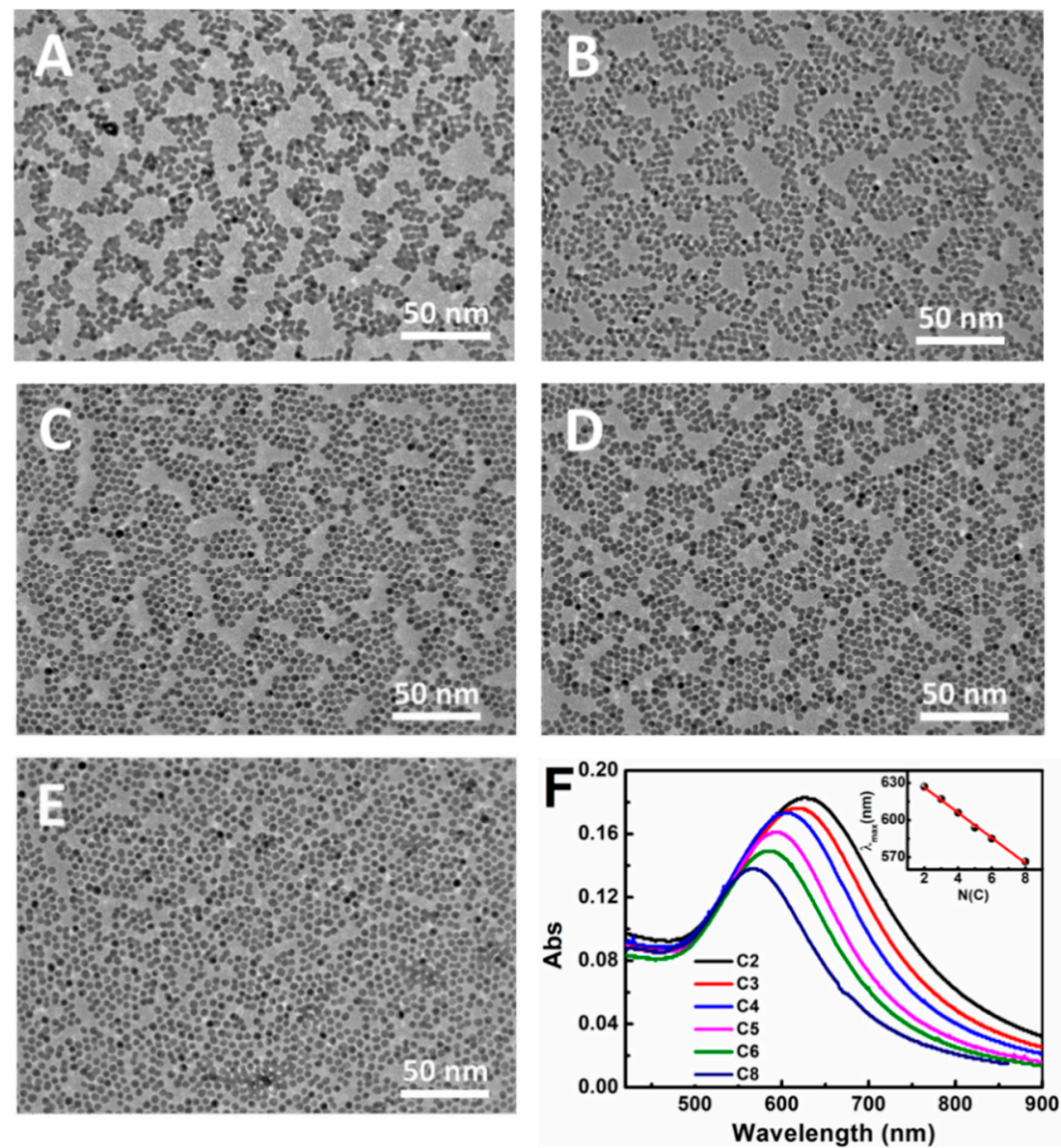

Figure 3. TEM images of the cross-linking monolayer prepared by the exchange reaction with different dithiol molecules: (A) 1,2-ethanedithiol; (B) 1,3-dimercaptopropane; (C) 1,4-butanedithiol; (D) 1,5-pentanedithiol; (E) 1,6-hexanedithiol; (F) UV-Vis absorption spectrum of the cross-linking monolayer with different dithiol molecules, inset is the plot of $\lambda_{\max } v s$ dithiol carbon numbers.

To prove that this ligand exchange strategy has a general applicability, a similar cross-linking reaction based on the Au NP array was performed on the Teflon substrate. As shown in Figure 4, the color of the Au NP array gradually changed from bluish violet to green with a shorter dithiol, indicating 
a variation in the LSPR of the Au NP array. The TEM characterization results also suggested that the array became more compact after the cross-linking reaction, although some damaged holes formed in the array structure (deriving from the dithiol molecule stretching for nanoparticles), as shown in Figure 5. Therefore, the above study results clearly show that the ligand exchange strategy is not only suitable for different ligands, but is also appropriate for other substrates supporting the Au NP array.
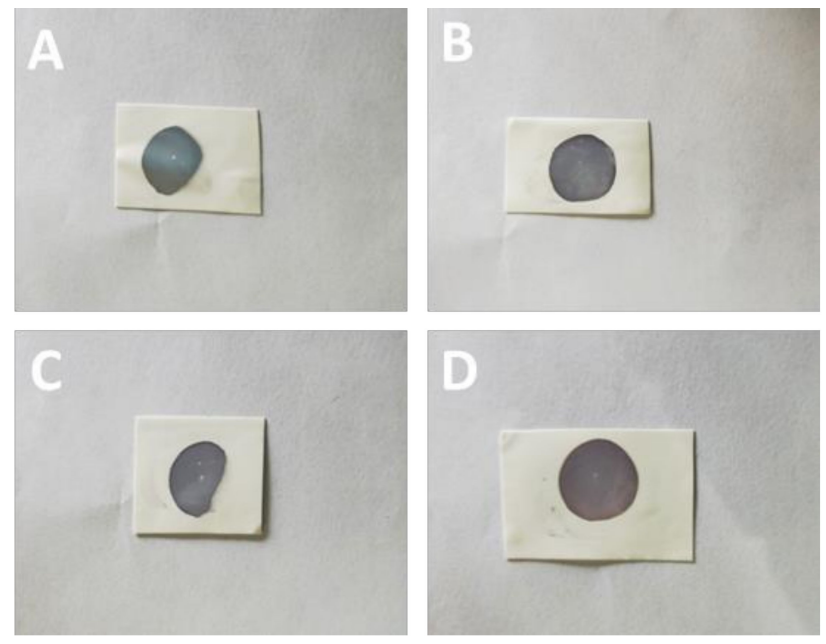

Figure 4. Photos of the Au NP array cross-linked with ligands on the Teflon substrate: (A) 1,2-ethanedithiol; (B) 1,4-butanedithiol; (C) 1,6-hexanedithiol; (D) 1,8-Octanedithiol.
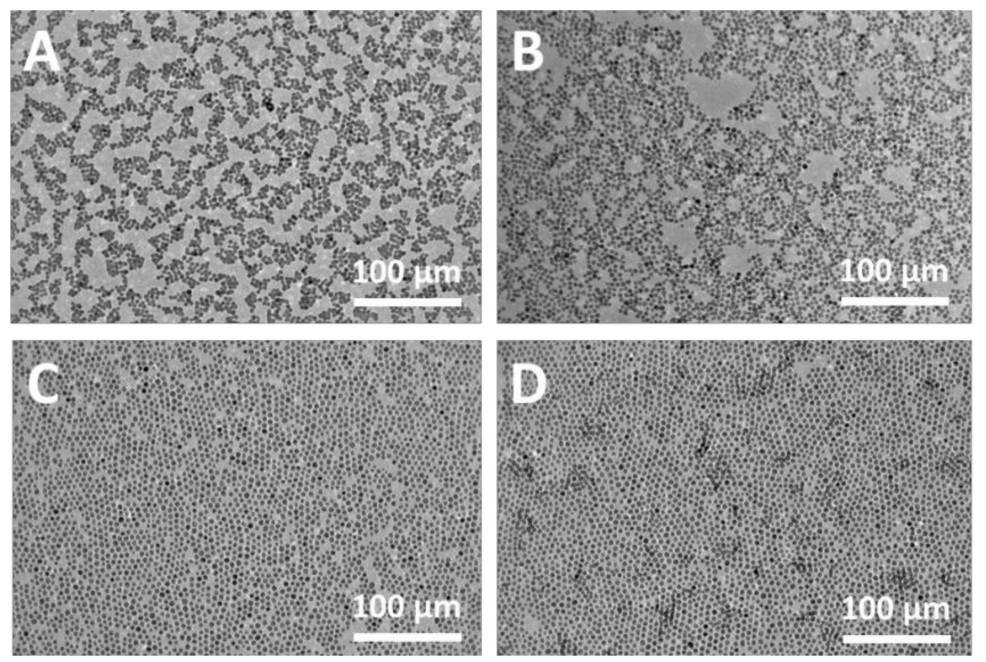

Figure 5. TEM images of the cross-linking monolayer array with different dithiol molecules on the Teflon substrate (A) 1,2-ethanedithiol; (B) 1,4-butanedithiol; (C) 1,6-hexanedithiol; (D) 1,8-octanedithiol.

Apparently, the cross-linking array with a different internal structure could be obtained by this liquid exchanging system. In order to precisely appraise the variation of the array structure, a 2D GISAXS measurement was performed. As shown in Figure 6, there were only two diffraction arcs along the in-plane direction, which suggests that the Au NPs assembled to form a well-organized array parallel to the substrate. Comparing the three patterns, their diffraction arcs had a significant difference along the q-direction. The arcs became more blurred and shifted toward a larger q value with a shorter cross-linking dithiol, indicating the reduced ordering degree and interparticle spacing in the array. To quantitatively study the variation of interparticle spacing, 1D GISAXS was also employed to investigate the array structure. As shown in Figure 6D, the scattering peak value increased and the intensity weakened along with the decreased carbon numbers. The variation of the interparticle spacing against the carbon numbers of the dithiol is shown in the inset of Figure 6D. The interparticle distance 
gradually decreased from $6.14 \mathrm{~nm}$ to $5.83 \mathrm{~nm}$ after cross-linking with different dithiol molecules $\left(\mathrm{C}_{2}-\mathrm{C}_{6}\right)$. These results sufficiently demonstrate that the interparticle spacing of the arrays could be regulated by variating the ligand length.
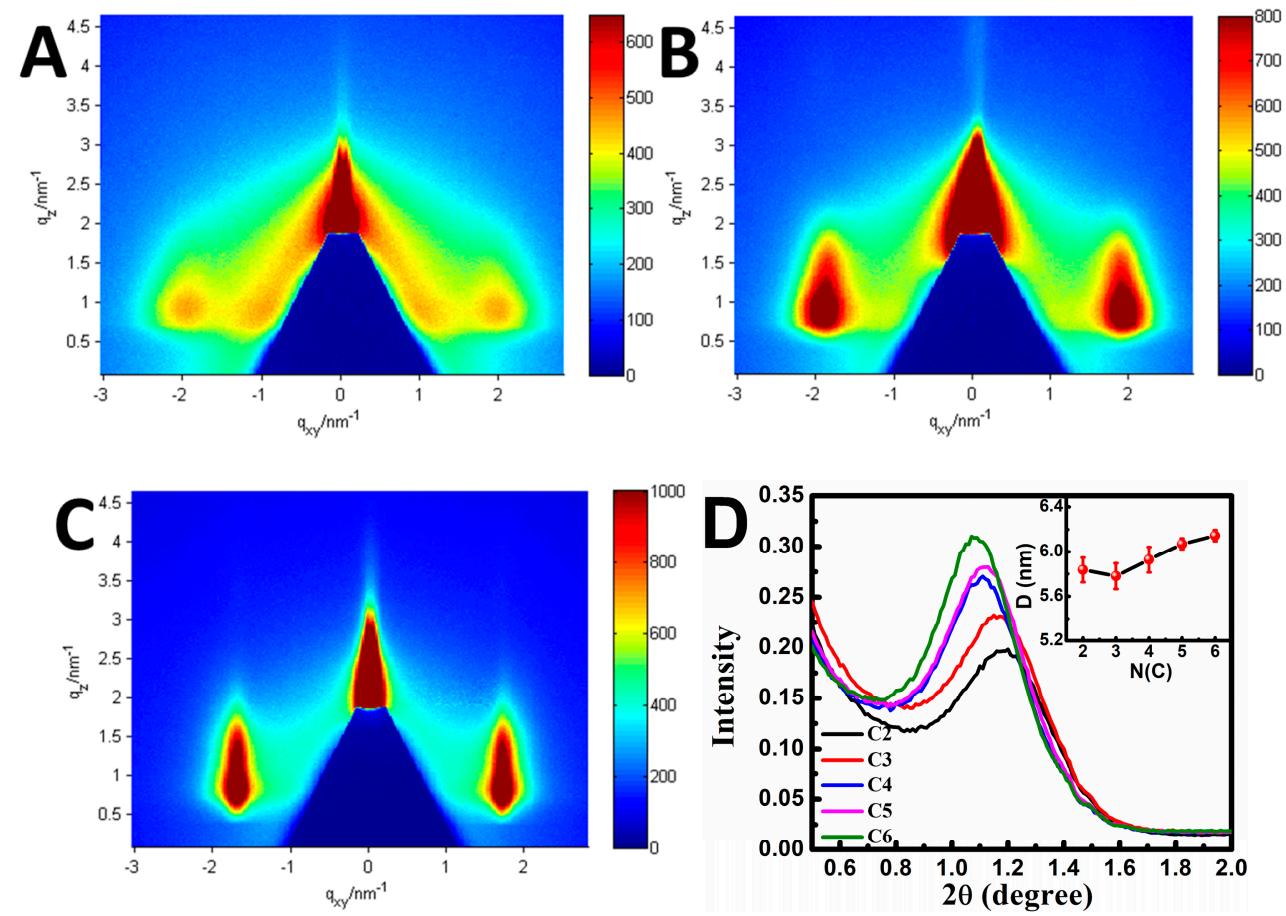

Figure 6. Two-dimensional GISAXS patterns obtained from an array cross-linked with (A) 1,2-ethanedithiol; (B) 1,4-butanedithiol; (C) 1,6-hexanedithiol. (D) Respective line cuts along the horizontal (qy) axis.

\subsection{Mechanical Properties of the Cross-Linking Monolayer Film}

As we all know, the mechanical properties of membranes are considerable for their application in various fields. For the self-assembled Au NP array, some non-covalent bonding forces (including van der Walls forces, electrostatic forces, etc.) dominate the main interactions between nanoparticles, which are relatively weak and easily influenced by the external surrounding. Here, the dithiol molecule linking Au NPs (Au-S bonding, the covalent bonding effect) could largely improve the interaction of adjacent nanoparticles. This generates a positive effect for the enhanced film strength. Although some studies have reported relevant work for strengthening the mechanical intensity of the membrane by the cross-linking reaction, systematic research on the modulus and cross-linking molecule has not been referred. Figure 7 shows the relationship between the ligand molecule length and Young's modulus for the cross-linking array. Obviously, the membrane modulus improved significantly with the more carbon numbers of dithiol. The Young's modulus of membrane $\left(\mathrm{E}_{\mathrm{f}}\right)$ is calculated according to the formula as follows:

$$
\mathrm{E}_{\mathrm{f}}=3 \mathrm{E}_{\mathrm{s}}\left(1-\mathrm{V}_{\mathrm{f}}^{2} / 1-\mathrm{V}_{\mathrm{s}}^{2}\right)(\mathrm{d} / 2 \pi \mathrm{h})^{3}
$$

where $E_{s}, V_{f}, V_{s}, d$, and $h$ represent the Young's modulus of the PDMS, Poisson ratios of the membrane and PDMS, bucking wavelength, and membrane thickness, respectively. The Young's modulus of membranes was calculated as $204.5 \mathrm{MPa}, 236 \mathrm{MPa}, 247.5 \mathrm{MPa}$, and $268.5 \mathrm{MPa}$, respectively, indicating an improvement in film mechanical property. We attribute this improved membrane strength to their microstructure. As discussed above, the cross-linking monolayer emerges with some broken holes in the array when using the short chain dithiol molecule as the cross-linking agent. This undesirable breakage generates a negative effect for the stability and strength of the membrane and finally presents the fragile property and weak strength. Therefore, the dithiol molecule with the appropriate carbon 
atom number is critical for its mechanical property. In our work, due to the approximate length between the dithiol chain length and interparticle spacing, the dithiol molecule with six carbon atoms cross-linking the nanoparticle array hardly changed the initial state of the array, which resulted in the integral structure was maintained as much as possible and the highest membrane strength (no damage in the structure). Here, the cross-linking technology is an available and useful strategy to strengthen the membranes' intensity, and also offers an understanding of the membranes' strengthening mechanism.

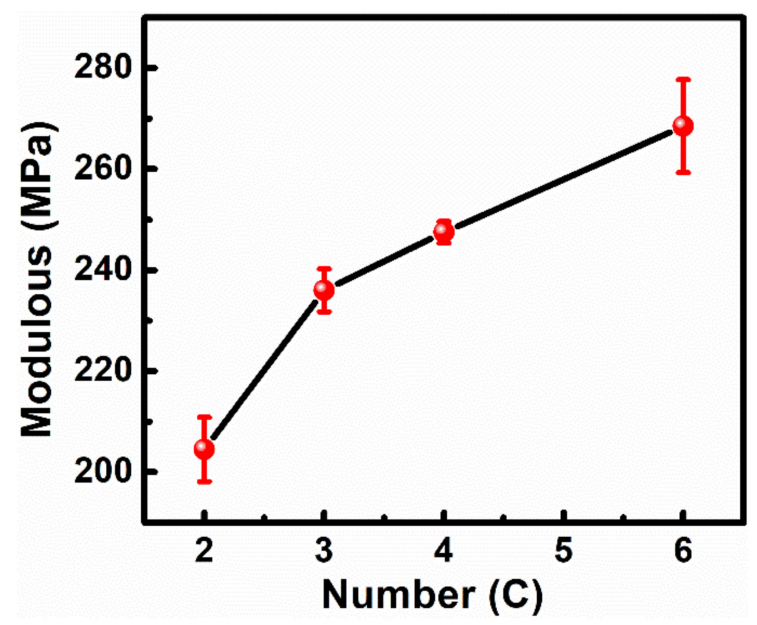

Figure 7. The Young's modulus of the cross-linking monolayer film vs. the carbon numbers of the dithiol molecule.

\section{Conclusions}

In summary, a cross-linking NP monolayer membrane was fabricated by the ligand exchange reaction. A series of dithiol molecules with different carbon numbers were used to replace the ligand molecule and successfully connected the adjacent nanoparticle, which led to the different microstructure of the nanoparticle monolayer. Additionally, this cross-linking manner exhibited a positive effect in enhancing the mechanical intensity of the membranes while having a general applicability for the other substrate. Moreover, we established a preliminary relationship between the ligands' length and the membranes' Young's modulus, which offers a great reference in future applications as nano-devices.

Author Contributions: Preparation of the sample, J.Z. and X.C. (Xiaoqing Cao); Characterization of sample, L.L. and X.C. (Xingcheng Cui); Writing-original draft preparation, J.Z. and X.C. (Xiaoqing Cao); Writing-review and editing, J.Z. and Y.F.

Funding: This work was supported by the National Natural Science Foundation of China (No. 51601032), the Fundamental Research Funds for the Central Universities (N170204015), and the Provincial Science and Technology Project/Doctor Start Fund (20170520385).

Conflicts of Interest: The authors declare no conflict of interest.

\section{References}

1. Schlicke, H.; Battista, D.; Kunze, S.; Schröter, C.J.; Eich, M.; Vossmeyer, T. Freestanding Membranes of Cross-Linked Gold Nanoparticles: Novel Functional Materials for Electrostatic Actuators. ACS Appl. Mater. Interfaces 2015, 7, 15123-15128. [CrossRef]

2. Schlicke, H.; Leib, E.W.; Petrov, A.; Schröder, J.H.; Vossmeyer, T. Elastic and viscoelastic properties of cross-linked gold nanoparticles probed by AFM bulge tests. J. Phys. Chem. C 2014, 118, 4386-4395. [CrossRef]

3. Shi, Q.; Gómez, D.E.; Dong, D.; Sikdar, D.; Fu, R.; Liu, Y.; Zhao, Y.; Smilgies, D.; Cheng, W. 2D Freestanding Janus Gold Nanocrystal Superlattices. Adv. Mater. 2019, 31, 1900989. [CrossRef]

4. Someya, T.; Sekitani, T.; Iba, S.; Kato, Y.; Kawaguchi, H.; Sakurai, T. A large-area, flexible pressure sensor matrix with organic field-effect transistors for artificial skin applications. Proc. Natl. Acad. Sci. USA 2004, 101, 9966-9970. [CrossRef] 
5. Wang, C.; Hwang, D.; Yu, Z.; Takei, K.; Park, J.; Chen, T.; Ma, B.; Javey, A. User-interactive electronic skin for instantaneous pressure visualization. Nat. Mater. 2013, 12, 899-904. [CrossRef]

6. Liu, Y.; Chen, H.; Xu, C.; Sun, Y.; Li, S.; Jiang, M.; Qin, G. Control of Catalytic Activity of Nano-Au through Tailoring the Fermi Level of Support. Small 2019, 15, 1901789. [CrossRef]

7. Wang, Y.; Cao, F.; Lin, W.; Zhao, F.; Zhou, J.; Li, S.; Qin, G. In situ synthesis of Ni/NiO composites with defect-rich ultrathin nanosheets for highly efficient biomass-derivative selective hydrogenation. J. Mater. Chem. A 2019, 7, 17834-17841. [CrossRef]

8. Cao, X.; Zhou, J.; Wang, H.; Li, S.; Wang, W.; Qin, G. Abnormal thermal stability of sub-10 nm Au nanoparticles and their high catalytic activity. J. Mater. Chem. A 2019, 7, 10980-10987. [CrossRef]

9. Li, S.; Cai, J.; Liu, Y.; Gao, M.; Cao, F.; Qin, G. Tuning orientation of doped hematite photoanodes for enhanced photoelectrochemical water oxidation. Sol. Energy Mater. Sol. Cells 2018, 179, 328-333. [CrossRef]

10. Pradhan, S.; Ghosh, D.; Xu, L.P.; Chen, S. Interparticle charge transfer mediated by $\pi-\pi$ stacking of aromatic moieties. J. Am. Chem. Soc. 2007, 129, 10622-10623. [CrossRef]

11. Zhou, J.; Liu, H.; Wang, T.; Li, Y.; Zhang, J.; Lu, Z.; Fu, Y.; Li, F. Adjusting the inter-particle spacing of a nanoparticle array at the sub-nanometre scale by thermal annealing. Chem. Commun. 2014, 50, 14547-14549. [CrossRef]

12. Edel, J.B.; Kornyshev, A.A.; Kucernak, A.R.; Urbakh, M. Fundamentals and applications of self-assembled plasmonic nanoparticles at interfaces. Chem. Soc. Rev. 2016, 45, 1581-1596. [CrossRef]

13. Markutsya, S.; Jiang, C.; Pikus, Y.; Tsukruk, V.V. Freely suspended layer-by-layer nanomembranes: Testing micromechanical properties. Adv. Funct. Mater. 2005, 15, 771-780. [CrossRef]

14. Min, Y.; Akbulut, M.; Kristiansen, K.; Golan, Y.; Israelachvili, J. The role of interparticle and external forces. Nat. Mater. 2008, 7, 527-538. [CrossRef]

15. Cheng, W.; Campolongo, M.J.; Tan, S.J.; Luo, D. Freestanding ultrathin nano-membranes via self-assembly. Nano Today 2009, 4, 482-493. [CrossRef]

16. Xia, H.; Wang, D. Fabrication of macroscopic freestanding films of metallic nanoparticle monolayers by interfacial self-assembly. Adv. Mater. 2008, 20, 4253-4256. [CrossRef]

17. Lin, Y.; Skaff, H.; Böker, A.; Dinsmore, A.D.; Emrick, T.; Russell, T.P. Ultrathin Cross-Linked Nanoparticle Membranes. J. Am. Chem. Soc. 2003, 125, 12690-12691. [CrossRef]

18. Le Ouay, B.; Guldin, S.; Luo, Z.; Allegri, S.; Stellacci, F. Freestanding Ultrathin Nanoparticle Membranes Assembled at Transient Liquid-Liquid Interfaces. Adv. Mater. Interfaces 2016, 3, 1600191. [CrossRef]

19. Schlicke, H.; Rebber, M.; Kunze, S.; Vossmeyer, T. Resistive pressure sensors based on freestanding membranes of gold nanoparticles. Nanoscale 2016, 8, 183-186. [CrossRef]

20. Schlicke, H.; Schröter, C.J.; Vossmeyer, T. Electrostatically driven drumhead resonators based on freestanding membranes of cross-linked gold nanoparticles. Nanoscale 2016, 8, 15880-15887. [CrossRef]

21. Kowalczyk, B.; Apodaca, M.M.; Nakanishi, H.; Smoukov, S.K.; Grzybowski, B.A. Lift-Off and Micropatterning of Mono- and Multilayer Nanoparticle Films. Small 2009, 5, 1970-1973. [CrossRef] [PubMed]

22. Luther, J.M.; Law, M.; Song, Q.; Perkins, C.L.; Beard, M.C.; Nozik, A.J. Structural, Optical, and Electrical Properties of Self-Assembled Films of PbSe Nanocrystals Treated with 1,2-Ethanedithiol. ACS Nano 2008, 2, 271-280. [CrossRef] [PubMed]

23. Tangirala, R.; Baker, J.L.; Alivisatos, A.P.; Milliron, D.J. Modular Inorganic Nanocomposites by Conversion of Nanocrystal Superlattices. Angew. Chemie Int. Ed. 2010, 49, 2878-2882. [CrossRef] [PubMed]

24. Zhou, J.; Ni, J.; Song, Y.; Chen, B.; Li, Y.; Zhang, Y.; Li, F.; Jiao, Y.; Fu, Y. Transfer of ordered nanoparticle array and its application in high-modulus membrane fabrication. J. Mater. Chem. C 2014, 2, 6410-6414. [CrossRef]

25. Zhou, J.; Song, G.; Li, Y.; Song, Y.; Chen, B.; Zhang, X.; Wang, T.; Fu, Y.; Li, F. Thermal Annealing: A Facile Way of Conferring Responsivity to Inert Alkyl-Chain-Passivated Nanoparticle Arrays. Langmuir 2014, 30, 13052-13057. [CrossRef]

26. Martin, M.N.; Basham, J.I.; Chando, P.; Eah, S.-K. Charged Gold Nanoparticles in Non-Polar Solvents: 10-min Synthesis and 2D Self-Assembly. Langmuir 2010, 26, 7410-7417. [CrossRef]

(C) 2019 by the authors. Licensee MDPI, Basel, Switzerland. This article is an open access article distributed under the terms and conditions of the Creative Commons Attribution (CC BY) license (http://creativecommons.org/licenses/by/4.0/). 